\title{
Better bounds on optimal measurement and entanglement recovery, with applications to uncertainty and monogamy relations
}

\author{
Joseph M. Renes \\ Institute for Theoretical Physics, ETH Zürich, Switzerland
}

\begin{abstract}
We extend the recent bounds of Sason and Verdú relating Rényi entropy and Bayesian hypothesis testing [arXiv:1701.01974] to the quantum domain and show that they have a number of different applications. First, we obtain a sharper bound relating the optimal probability of correctly distinguishing elements of an ensemble of states to that of the pretty good measurement, and an analogous bound for optimal and pretty good entanglement recovery. Second, we obtain bounds relating optimal guessing and entanglement recovery to the fidelity of the state with a product state, which then leads to tight tripartite uncertainty and monogamy relations.
\end{abstract}

\section{Introduction}

Successful analysis of information processing protocols requires suitable measures of information and entropy, particularly those that satisfy the data processing inequality, the statement that a formal measure of information satisfies the intuitive requirement that a noisy channel cannot increase it. One broad class of measures is given by the Rényi divergences, which includes the usual Shannon and von Neumann definitions of mutual information and entropy. But even more, the Rényi divergences also encompass optimal and "pretty good" strategies for distinguishing quantum states or recovering entanglement, and are related to the oft-used fidelity function. Hence new insights into these measures often leads to new results for these operational tasks. This is the case in [1], for instance, which found new conditions for the optimality of the pretty good measurement by investigating the relationship of various quantum Rényi divergences.

Here we extend a recent result by Sason and Verdú [2], which establishes a whole class of Fano-like inequalities involving the Rényi divergence and optimal distinguishing probability, to the quantum domain. Though the inequalities are essentially an immediate consequence of the data processing inequality, they turn out to have a number of interesting applications. First, we find improved bounds relating the pretty good measurement to the optimal measurement, as well as analogous bounds for pretty good and optimal entanglement recovery. Second, by establishing a new relation between the optimal guessing probability and the fidelity, we can provide a complete characterization of the set of admissible guessing probabilities in an uncertainty game [3, 4], which is also related to wave-particle duality relations in multiport interferometers [5]. The goal of game is to provide predictions of the values of potential measurements of two conjugate observables on a quantum system; the uncertainty principle implies that the predictions cannot both be accurate. The same relation holds for fidelity and optimal entanglement recovery, and in this context gives a complete characterization of the possible entanglement fidelities two different parties can have with a common system, resolving a conjecture for the "singlet monogamy" studied in [6].

\section{Setup}

\subsection{Rényi divergences}

For two classical distributions $P$ and $Q$, the Rényi divergence of order $\alpha \in(0,1) \cup(1, \infty)$ is given by [7]

$$
D_{\alpha}(P, Q):=\frac{1}{\alpha-1} \log \sum_{x} P(x)^{\alpha} Q(x)^{1-\alpha} .
$$

The limits $\alpha=0,1, \infty$ are obtained by continuity in $\alpha$, which gives $D_{0}(P, Q)=-\log \sum_{x: P(x)>0} Q(x), D_{1}(P, Q)=$ $\sum_{x} P(x) \log \frac{P(x)}{Q(x)}$, and $D_{\infty}(P, Q)=\max _{x} \log \frac{P(x)}{Q(x)}$. In the case of distributions on a binary-valued random variable it will be convenient to define the binary Rényi divergence

$$
d_{\alpha}(p, q):=\frac{1}{\alpha-1} \log \left(p^{\alpha} q^{1-\alpha}+(1-p)^{\alpha}(1-q)^{1-\alpha}\right),
$$

where $0 \leq p \leq 1$ and $0 \leq q \leq 1$. Again the limiting cases are established by continuity.

In the quantum case there are several possible definitions of the Rényi divergence, due to the choices of ordering the density operators. Only those that satisfy the data processing inequality are useful for our purposes, and there are several; see [8] for an overview. Here we will focus on the minimal, or sandwiched, Rényi divergence $[9,10]$,

$$
D_{\alpha}(\rho, \sigma):=\frac{1}{\alpha-1} \log \operatorname{Tr}\left[\left(\sigma^{\frac{1-\alpha}{2 \alpha}} \rho \sigma^{\frac{1-\alpha}{2 \alpha}}\right)^{\alpha}\right]
$$


The name "minimal" comes from the fact that quantity is the smallest in a family of possible Rényi divergences which satisfies the data processing inequality (in this case, for $\alpha \in\left[\frac{1}{2}, \infty\right]$ ) [8].

Though we will not explicitly make use of it here, we mention that the Rényi divergence can be used to define a conditional entropy by either $H_{\alpha}^{\downarrow}(X \mid B)_{\rho}:=\log |X|-D_{\alpha}\left(\rho_{X B}, \pi_{X} \otimes \rho_{B}\right)$ or $H_{\alpha}^{\uparrow}(X \mid B)_{\rho}:=\sup _{\sigma}(\log |X|-$ $D_{\alpha}\left(\rho_{X B}, \pi_{X} \otimes \sigma_{B}\right)$. In the classical case this definition goes back to Arimoto [11] and appears to be the most meaningful extension of the usual Shannon conditional entropy to the Rényi setting [12, 13].

\subsection{Guessing probabilities and entanglement recovery}

The minimal divergence is also interesting due to its connections with the fidelity function as well as optimal and "pretty good" guessing probabilities and entanglement recovery. For $F(\rho, \sigma)=\|\sqrt{\rho} \sqrt{\sigma}\|_{1}$ the fidelity of the states $\rho$ and $\sigma$, we have $D_{1 / 2}(\rho, \sigma)=-\log F(\rho, \sigma)^{2}$.

An arbitrary ensemble of states $\varphi_{x}$ with prior probabilities $p_{x}$ can be encapsulated in the classical-quantum state $\rho_{X B}=\sum_{x} p_{x}|x\rangle\left\langle\left. x\right|_{x} \otimes\left(\varphi_{x}\right)_{B}\right.$. Any given measurement $\Lambda$ on $B$ results in some average probability of correctly guessing, $P(X \mid B)_{\rho, \Lambda}=\sum_{x} p_{x} \operatorname{Tr}\left[\varphi_{x} \Lambda_{x}\right]$. As shown in [14], the optimal probability $P_{\text {opt }}$ satisfies

$$
\inf _{\sigma} D_{\infty}\left(\rho_{X B}, \pi_{X} \otimes \sigma_{B}\right)=\log |X| P_{\text {opt }}(X \mid B)_{\rho},
$$

where $\pi_{X}$ is the completely mixed state (uniform distribution). The "pretty good measurement" $[15,16]$ uses the POVM elements $\Lambda_{x}=\varphi^{-1 / 2} p_{x} \varphi_{x} \varphi^{-1 / 2}$ for $\varphi=\sum_{x} p_{x} \varphi_{x}$, and its guessing probability $P_{\mathrm{pg}}$ satisfies [17]

$$
D_{2}\left(\rho_{X B}, \pi_{X} \otimes \rho_{B}\right)=\log |X| P_{\mathrm{pg}}(X \mid B)_{\rho} .
$$

When the $\varphi_{x}$ commute and $B$ is effectively a classical random variable $Y$, the pretty good measurement reduces to guessing $X$ by sampling from the distribution $P_{X \mid Y=y}$ for the observed value of $Y$. Beyond its use in quantum information theory, this measurement has also been used to construct decoders for error-correcting codes in classical information theory [18].

The fully quantum analog of the guessing scenario is that of entanglement recovery by local action. For an arbitrary bipartite entangled state $\rho_{A B}$. a quantum channel $\mathcal{E}_{A^{\prime} B}$ taking $B$ to $A^{\prime} \simeq A$ results in some (squared) fidelity with the maximally entangled state $|\Phi\rangle_{A A^{\prime}}=\frac{1}{\sqrt{|A|}} \sum_{x}|x\rangle_{A}|x\rangle_{A^{\prime}}, R(A \mid B)_{\rho, \mathcal{E}}=\operatorname{Tr}\left[\Phi_{A^{\prime}} \mathcal{E}_{A^{\prime} \mid B}\left(\rho_{A B}\right)\right]$. The optimal fidelity $R_{\text {opt }}$ satisfies [14]

$$
\inf _{\sigma} D_{\infty}\left(\rho_{A B}, \pi_{A} \otimes \sigma_{B}\right)=\log |A|^{2} R_{\text {opt }}(A \mid B)_{\rho} .
$$

The "pretty good recovery" uses the map $\mathcal{E}_{A^{\prime} \mid B}\left(\sigma_{A B}\right)=\operatorname{Tr}_{B}\left[\rho_{B}^{-1 / 2} \rho_{A^{\prime} B} \rho_{B}^{-1 / 2} \sigma_{A B}^{T_{B}}\right]$ and satisfies [19]

$$
D_{2}\left(\rho_{A B}, \pi_{A} \otimes \rho_{B}\right)=\log |A|^{2} R_{\mathrm{pg}}(A \mid B)_{\rho} .
$$

When $\operatorname{Tr}_{B}\left[\rho_{A B}\right]=\pi_{A}$, this is the recovery map of $[20]$.

\section{Bounds}

Regarding the POVM $\left\{\Lambda_{x}\right\}$ as the quantum-classical channel $\mathcal{M}_{X^{\prime} \mid B}$, we can express the guessing probability as $P(X \mid B)_{\rho, \Lambda}=\operatorname{Tr}\left[\Pi_{X X^{\prime}} \mathcal{M}_{X^{\prime} \mid B}\left(\rho_{X B}\right)\right]$, where $\Pi_{X X^{\prime}}=\sum_{x \in \mathcal{X}}|x\rangle\left\langle\left. x\right|_{X} \otimes \mid x\right\rangle\left\langle\left. x\right|_{X^{\prime}}\right.$. Note that $\operatorname{Tr}\left[\Pi_{X X^{\prime}} \mathcal{M}_{X^{\prime} \mid B}\left(\pi_{X} \otimes\right.\right.$ $\left.\left.\sigma_{B}\right)\right]=\frac{1}{|X|}$ for any state $\sigma_{B}$. The projector $\Pi_{X X^{\prime}}$ is part of a two-outcome measurement, a test, described by the channel $\mathcal{T}_{Y \mid X X^{\prime}}$. The random variable $Y$ equals 1 when the test passes, corresponding to $\Pi_{X X^{\prime}}$, and zero if it fails, corresponding to $\mathbb{1}_{X X^{\prime}}-\Pi_{X X^{\prime}}$. Similarly, the expression for $R(A \mid B)_{\rho, \mathcal{E}}$ makes use of the test $\mathcal{T}_{Y \mid A A^{\prime}}$ involving $\Phi_{A A^{\prime}}$. And in this case we have, for any $\sigma_{A^{\prime}}, \operatorname{Tr}\left[\Phi_{A A^{\prime}} \pi_{A} \otimes \sigma_{A^{\prime}}\right]=\frac{1}{|A|^{2}}$. Applying the data processing inequality of the Rényi divergence for $\mathcal{T} \circ \mathcal{M}$ or $\mathcal{T} \circ \mathcal{E}$ immediately gives our main result.

Proposition 1. Let $\sigma_{B}$ be any normalized state and $\alpha \in\left[\frac{1}{2}, \infty\right]$. For arbitrary classical-quantum states $\rho_{X B}$ and measurements $\left\{\Lambda_{x}\right\}$ on $B$, we have

$$
D_{\alpha}\left(\rho_{X B}, \pi_{X} \otimes \sigma_{B}\right) \geq d_{\alpha}\left(P(X \mid B)_{\rho, \Lambda}, \frac{1}{|X|}\right) .
$$

For $\rho_{A B}$ an arbitrary bipartite quantum state and $\mathcal{E}_{A^{\prime} \mid B}$ a quantum channel from $B$ to $A^{\prime} \simeq A$, we have

$$
D_{\alpha}\left(\rho_{A B}, \pi_{A} \otimes \sigma_{B}\right) \geq d_{\alpha}\left(R(A \mid B)_{\rho, \mathcal{E}}, \frac{1}{|A|^{2}}\right) .
$$
ties

Choosing $\alpha=1, \sigma_{B}=\rho_{B}$, and the optimal measurement $\Lambda$ or recovery map $\mathcal{E}_{A^{\prime} \mid B}$ gives the Fano inequali-

$$
\begin{aligned}
H(X \mid B)_{\rho} & \leq\left(1-P_{\mathrm{opt}}(X \mid B)_{\rho}\right) \log (|X|-1)+h_{2}\left(P_{\mathrm{opt}}(X \mid B)_{\rho}\right), \quad \text { and } \\
H(A \mid B)_{\rho} & \leq-\log |A|+\left(1-R_{\mathrm{opt}}(A \mid B)_{\rho}\right) \log \left(|A|^{2}-1\right)+h_{2}\left(R_{\mathrm{opt}}(A \mid B)_{\rho}\right),
\end{aligned}
$$

where $h_{2}(x)=-x \log _{2} x-(1-x) \log _{2}(1-x)$ is the binary entropy. 


\subsection{Pretty good measurement and entanglement recovery}

Choosing $\mathcal{M}_{X^{\prime} \mid Y}$ to be the optimal measurement for given $\rho_{X B}$ and using (5) in (8) gives

$$
P_{\mathrm{pg}}(X \mid B)_{\rho} \geq P_{\mathrm{opt}}(X \mid B)_{\rho}^{2}+\frac{\left(1-P_{\mathrm{opt}}(X \mid B)_{\rho}\right)^{2}}{|X|-1} .
$$

In the classical case this was first shown by [21, Theorem 3], though without the connection between $D_{2}$ and $P_{\mathrm{pg}}(X \mid B)_{\rho}$.

Equality can be attained (also shown in [21]), as illustrated by the "L distribution" with weight $p_{0}$ on $X=0$ and $\left(1-p_{0}\right) /(|X|-1)$. The optimal guess is always $X=0$, meaning $P_{\mathrm{opt}}(X)=p_{0}$. Meanwhile, the pretty good measurement generates its guess in this case by sampling from the distribution. Therefore $P_{\mathrm{pg}}(X)=p_{0}^{2}+(|X|-1)\left(\frac{1-p_{0}}{|X|-1}\right)^{2}$, which is precisely the righthand side above.

Taking the limit $|X| \rightarrow \infty$, we recover the previously-known result, $P_{\mathrm{pg}}(X \mid B)_{\rho} \geq P_{\mathrm{opt}}(X \mid B)_{\rho}^{2}$, first shown in [22] in the classical case (again, just as a statement involving on $D_{2}$ ) and in [20] in the quantum case. The new bound resolves a defect of the previous bound, in that the value of the new bound is always larger than $1 /|X|$. To see this, observe that the righthand side of (12) minus $1 /|X|$ is simply $\frac{\left(|X| P_{\text {of }}\right)^{2}}{|X|(X \mid-1)}>0$. This ensures that the bound is meaningful for any value of $P_{\text {opt }}(X \mid B)_{\rho}$, whereas the previous bound is only meaningful when $P_{\text {opt }}(X \mid B)_{\rho} \geq 1 / \sqrt{|X|}$.

Choosing $\mathcal{E}_{A^{\prime} \mid B}$ to be the optimal recovery map for given $\rho_{A B}$ and using (7) in (9) similarly gives

$$
R_{\mathrm{pg}}(A \mid B)_{\rho} \geq R_{\mathrm{opt}}(A \mid B)_{\rho}^{2}+\frac{\left(1-R_{\mathrm{opt}}(A \mid B)_{\rho}\right)^{2}}{|A|^{2}-1} .
$$

Equality can also be attained in this bound, by essentially the same example. Suppose $\rho_{A B}$ is a Bell-diagonal state with weight $p_{0}$ on $|\Phi\rangle$ and $1-p_{0}$ evenly spread over the remaining $|A|^{2}-1$ Bell states. The local state on system $B$ is the same for all Bell states, so there is no advantage to applying a nontrivial recovery map on $B$; hence $R_{\text {opt }}(A \mid B)_{\rho}=p_{0}$. On the other hand, using the pretty good recovery leads to $R_{\mathrm{pg}}(A \mid B)_{\rho}=\operatorname{Tr}\left[\rho_{A B}^{2}\right]$, which then gives the righthand side.

Bounds in the other direction can be obtained by choosing $\mathcal{M}$ to be the pretty good measurement and using (4) in (8), or $\mathcal{E}$ to be the pretty good recovery and using (6) in (9). However, this leads back to the obvious lower bounds $P_{\text {opt }}(X \mid B)_{\rho} \geq P_{\mathrm{pg}}(X \mid B)_{\rho}$ and $R_{\text {opt }}(A \mid B)_{\rho} \geq R_{\mathrm{pg}}(A \mid B)_{\rho}$.

\subsection{Uncertainty and monogamy relations}

Again choosing the optimal measurement or recovery map but now using the relationship between $D_{1 / 2}$ and the fidelity gives

$$
\begin{aligned}
& F\left(\rho_{X B}, \pi_{X} \otimes \sigma_{B}\right)^{2} \leq \frac{1}{|X|}\left(\sqrt{P_{\mathrm{opt}}(X \mid B)_{\rho}}+\sqrt{|X|-1} \sqrt{1-P_{\mathrm{opt}}(X \mid B)_{\rho}}\right)^{2}, \\
& F\left(\rho_{A B}, \pi_{A} \otimes \sigma_{B}\right)^{2} \leq \frac{1}{|A|^{2}}\left(\sqrt{R_{\mathrm{opt}}(A \mid B)_{\rho}}+\sqrt{|A|^{2}-1} \sqrt{1-R_{\mathrm{opt}}(A \mid B)_{\rho}}\right)^{2} .
\end{aligned}
$$

In the case of classical $B$ the former bound was reported by Sason and Verdú [2, Equation 109]. Employing the "L distribution" again yields equality in both. Thus, the former is necessarily stronger than the bound reported by the author in [4, Equation 23] as well the bound discovered by Coles [5, Equation 6],

We can use (14) to completely characterize the region of allowed guessing probabilities in the three party uncertainty game considered in [4]. Suppose $\rho_{A B C}$ is a tripartite quantum state and $\psi_{X B}$ is the classicalquantum state resulting from measuring an observable $X$ on system $A$ and ignoring $C$, while $\xi_{Z C}$ is the classical-quantum state resulting from measuring the conjugate observable $Z$ on $A$ and ignoring $B$. An immediate question is what are the allowed values of $P(X \mid B)_{\psi, \Lambda}$ and $P(Z \mid C)_{\xi, \Gamma}$. To determine the boundary of the set, start with the uncertainty relation for min and max entropy [23], which can be expressed as $\max _{\sigma} F\left(\psi_{X B}, \pi_{X} \otimes \sigma_{B}\right)^{2} \geq P_{\text {opt }}(Z \mid C)_{\xi}$. Combining this with (14) gives

$$
|A| P_{\mathrm{opt}}(Z \mid C)_{\xi} \leq\left(\sqrt{P_{\mathrm{opt}}(X \mid B)_{\psi}}+\sqrt{|A|-1} \sqrt{1-P_{\mathrm{opt}}(X \mid B)_{\psi}}\right)^{2} .
$$

In principle, we could also interchange the two guessing probabilities to obtain another bound, but in fact this leads back to the same inequality. The bound also tightens the relation between fringe visibility and path 
distinguishability in symmetric multipath interferometers, Theorem 1 of [5], as these quantities are rescaled versions of the two guessing probabilities.

Equality can be attained in (16) by, unsurprisingly, a state involving an "L distribution". In particular, consider the case of trivial $B$ and $C$, and $|\theta\rangle_{A}$ the state with amplitudes $\sqrt{p_{0}}$ for $|0\rangle$ and $\sqrt{\frac{1-p_{0}}{|A|-1}}$ for $|x\rangle$ with $x \in\{1, \ldots,|A|-1\}$. Always guessing $X=0$ and $Z=0$ leads to equality. That this is optimal is to be expected, as it is easily seen that the state is a superposition of $X=0$ and $Z=0$ eigenstates. Thus the question of determining the region of allowed guessing probabilities, raised in [4], is completely solved.

Moreover, (16) has an elegant geometric interpretation. Letting $m=|A|, x=P_{\mathrm{opt}}(X \mid B)_{\psi}$, and $z=$ $P_{\text {opt }}(Z \mid C)_{\xi}$, it can be easily verified that the boundary is that of the ellipse

$$
\frac{(x+z-1)^{2}}{1 / m}+\frac{(x-z)^{2}}{(m-1) / m}=1
$$

in the region $1 / m \leq x, z \leq 1$. For arbitrary $m$, these are precisely the ellipses that just fit in the unit square.

Analogously to the use of (14) in the guessing game, (15) implies a bound on monogamy of entanglement; specifically, on the possible values of $R(A \mid B)_{\rho, \mathcal{E}}$ and $R(A \mid C)_{\rho, \mathcal{E}^{\prime}}$ for an arbitrary tripartite state $\rho_{A B C}$. In this case, using the duality of min and max entropy [14], which can be expressed as $\max _{\sigma} F\left(\rho_{A C}, \pi_{A} \otimes \sigma_{C}\right)^{2}=R(A \mid B)_{\rho}$, we obtain

$$
|A|^{2} R_{\text {opt }}(A \mid C)_{\rho} \leq\left(\sqrt{R_{\text {opt }}(A \mid B)_{\rho}}+\sqrt{|A|^{2}-1} \sqrt{1-R_{\text {opt }}(A \mid B)_{\rho}}\right)^{2} .
$$

Equality can be attained by a superposition of entanglement with $B$ and entanglement with $C$, namely $|\Psi\rangle_{A B C}=N^{-1 / 2}\left(\cos \theta|\Phi\rangle_{A B}|0\rangle_{C}+\sin \theta|\Phi\rangle_{A C}|0\rangle_{B}\right)$, with the normalization constant $N=1+\sin 2 \theta / d$ for $d=|A|$. Choosing trivial recovery maps, we obtain $R(A \mid B)_{\Psi \mathcal{I}}=(d \cos \theta+\sin \theta)^{2} /(d(d+\sin 2 \theta))$ and $R(A \mid C)_{\Psi, \mathcal{I}}=(d \sin \theta+\cos \theta)^{2} /(d(d+\sin 2 \theta))$. Comparing (18) and (16), it is apparent that the latter satisfies the ellipse equation with $x=R(A \mid B)_{\Psi, \mathcal{I}}, z=R(A \mid C)_{\Psi, \mathcal{I}}$, and $m=d^{2}$. It is then straightforward to check that the particular values of $R(A \mid B)_{\Psi, \mathcal{I}}$ and $R(A \mid C)_{\Psi, \mathcal{I}}$ satisfy (17).

In fact, $|\Psi\rangle$ was used in [6] to investigate the limits of what they term "singlet monogamy" and its relation to optimal cloning. The scenario they consider is nearly the same as here, except that the optimal channel in the recovery operation is restricted to be unitary and, importantly, they also consider monogamy involving more than two auxiliary parties. The state $|\Psi\rangle$ was conjectured to give the optimal bound, their Equation 6 , and the above derivation shows that their conjecture holds true for monogamy relations of three systems.

\section{Discussion and open problems}

By simple application of the data processing inequality, we have given new and useful bounds involving fidelity and guessing probabilities of optimal and pretty good measurements, as well as for the corresponding quantities for entanglement recovery. These allow the complete characterization of the allowed guessing probababilities when two different parties try to simultaneously predict the value of one of two conjugate measurements on a quantum system, as well as an analogous statement for the allowed entanglement fidelities two parties can each locally create with a common system.

It would be interesting to determine if the Rényi divergence at orders besides $\alpha=2, \infty$ is related to other particular guessing or entanglement recovery strategies, as this would immediately give new bounds. We can report the following partial result for $\alpha=3$ and the "quadratically-weighted" variant of the pretty good measurement, i.e. using $\Lambda_{x}=\bar{\varphi}^{-1 / 2} p_{x}^{2} \varphi_{x}^{2} \bar{\varphi}^{-1 / 2}$, for $\bar{\varphi}^{-1 / 2}=\sum_{x} p_{x}^{2} \varphi_{x}^{2}$ (discussed, e.g. in [24, Section 2.2]). Suppose the $\varphi_{x}$ all commute, so $B$ is essentially a classical random variable $Y$. Then the average guessing probability in this case is $P_{\text {quad }}(X \mid Y)_{\rho}=\sum_{y}\left(\sum_{x} P_{X Y}(x, y)^{3}\right) /\left(\sum_{x^{\prime}} P_{X Y}\left(x^{\prime}, y\right)^{2}\right)$. Bounding the denominator from above by $\left(\sum_{x^{\prime}} P_{X Y}\left(x^{\prime}, y\right)\right)^{2}$, one finds that $D_{3}\left(\rho_{X B}, \pi_{X} \otimes \rho_{B}\right) \leq \frac{1}{2} \log |X|^{2} P_{\text {quad }}(X \mid B)$. Employing (8) with $\alpha=3$ yields the relation

$$
P_{\text {quad }}(X \mid Y)_{\rho} \geq P_{\text {opt }}(X \mid Y)^{3}+\frac{\left(1-P_{\text {opt }}(X \mid Y)\right)^{3}}{(|X|-1)^{2}} .
$$

Unfortunately, this is weaker than the bound $P_{\text {quad }}(X \mid Y)_{\rho} \geq P_{\text {opt }}(X \mid Y)_{\rho}^{2}$ shown in [25, Theorem 10] and also valid for non-commuting ensembles. Nonetheless, this approach can presumably be easily extended to higher weights, e.g. cubic as considered in [26], and may prove useful there. One might also relate particular measurement strategies to other particular choices of the second argument to the divergence and investigate the implications of the data processing inequality in that context. For instance, [27, Theorem 4] shows that $\log |X| P_{\mathrm{pg}}(X \mid B)_{\rho} \geq D_{2}\left(\rho_{X B}, \frac{1}{|X|} \rho_{X B}+\left(1-\frac{1}{|X|}\right) \rho_{X} \otimes \rho_{B}\right)$.

Acknowledgments. I thank Raban Iten for useful conversations. This work was supported by the Swiss National Science Foundation (SNSF) via the National Centre of Competence in Research "QSIT". 


\section{References}

[1] R. Iten, J. Renes, and D. Sutter, "Pretty good measures in quantum information theory", IEEE Transactions on Information Theory 63, 1270-1279 (2017), arXiv:1608.08229 [quant-ph].

[2] I. Sason and S. Verdú, "Arimoto-Rényi Conditional Entropy and Bayesian $M$-ary Hypothesis Testing", (2017), arXiv:1701.01974 [cs.IT].

[3] M. Berta, M. Christandl, R. Colbeck, J. M. Renes, and R. Renner, "The uncertainty principle in the presence of quantum memory", Nature Physics 6, 659-662 (2010), arXiv:0909.0950 [quant-ph].

[4] J. M. Renes, "Uncertainty relations and approximate quantum error correction", Physical Review A 94, 032314 (2016), arXiv:1605.01420 [quant-ph].

[5] P. J. Coles, "Entropic framework for wave-particle duality in multipath interferometers", Physical Review A 93 , 062111 (2016), arXiv:1512.09081 [quant-ph].

[6] A. Kay, D. Kaszlikowski, and R. Ramanathan, "Optimal Cloning and Singlet Monogamy", Physical Review Letters 103, 050501 (2009), arXiv:0901.3626 [quant-ph].

[7] A. Rényi, "On Measures of Entropy and Information", in Proceedings of the Fourth Berkeley Symposium on Mathematical Statistics and Probability, Volume 1: Contributions to the Theory of Statistics (1961), pp. 547-561.

[8] M. Tomamichel, Quantum Information Processing with Finite Resources, Vol. 5, SpringerBriefs in Mathematical Physics (Springer International Publishing, Cham, 2016), arXiv:1504.00233 [quant-ph].

[9] M. Müller-Lennert, F. Dupuis, O. Szehr, S. Fehr, and M. Tomamichel, "On quantum Rényi entropies: A new generalization and some properties", Journal of Mathematical Physics 54, 122203 (2013), arXiv:1306.3142 [quant-ph].

[10] M. M. Wilde, A. Winter, and D. Yang, "Strong Converse for the Classical Capacity of Entanglement-Breaking and Hadamard Channels via a Sandwiched Rényi Relative Entropy", Communications in Mathematical Physics 331, 593622 (2014), arXiv:1306.1586 [quant-ph].

[11] S. Arimoto, "Information measures and capacity of order $\alpha$ for discrete memoryless channels", in Topics in Information Theory - 2nd Colloquium, Vol. 16, Colloquia Mathematica Societatis János Bolyai (1977), pp. 41-52.

[12] A. Teixeira, A. Matos, and L. Antunes, "Conditional Rényi Entropies", IEEE Transactions on Information Theory 58, 4273-4277 (2012).

[13] S. Fehr and S. Berens, "On the Conditional Rényi Entropy", IEEE Transactions on Information Theory 60, 6801-6810 (2014).

[14] R. König, R. Renner, and C. Schaffner, "The Operational Meaning of Min- and Max-Entropy”, IEEE Transactions on Information Theory 55, 4337-4347 (2009), arXiv:0807.1338 [quant-ph].

[15] V. P. Belavkin, "Optimal multiple quantum statistical hypothesis testing”, Stochastics 1, 315 (1975).

[16] P. Hausladen and W. K. Wootters, "A 'Pretty Good' Measurement for Distinguishing Quantum States”, Journal of Modern Optics 41, 2385 (1994).

[17] H. Buhrman, M. Christandl, P. Hayden, H.-K. Lo, and S. Wehner, "Possibility, impossibility, and cheat sensitivity of quantum-bit string commitment”, Physical Review A 78, 022316 (2008), arXiv:1302.5902 [quant-ph].

[18] M. H. Yassaee, M. R. Aref, and A. Gohari, "A technique for deriving one-shot achievability results in network information theory", in 2013 IEEE International Symposium on Information Theory (July 2013), pp. 1287-1291, arXiv:1303.0696 [cs.IT].

[19] M. Berta, P. J. Coles, and S. Wehner, "Entanglement-assisted guessing of complementary measurement outcomes", Physical Review A 90, 062127 (2014), arXiv:1302.5902 [quant-ph].

[20] H. Barnum and E. Knill, "Reversing quantum dynamics with near-optimal quantum and classical fidelity", Journal of Mathematical Physics 43, 2097-2106 (2002), arXiv:quant-ph/0004088.

[21] P. A. Devijver, "On a New Class of Bounds on Bayes Risk in Multihypothesis Pattern Recognition", IEEE Transactions on Computers C-23, 70-80 (1974).

[22] I. Vajda, "Bounds on the minimal error probability on checking a finite or countable number of hypotheses", Problems of Information Transmission 4, 9-19 (1968).

[23] M. Tomamichel and R. Renner, "Uncertainty Relation for Smooth Entropies", Physical Review Letters 106, 110506 (2011), arXiv:1009.2015 [quant-ph].

[24] V. P. Belavkin and V. P. Maslov, "Design of Optimal Dynamic Analyzers: Mathematical Aspects of Wave Pattern Recognition", in Mathematical Aspects of Computer Engineering (Mir, Moscow, 1987), pp. 146-237, arXiv:quant-ph/0412031.

[25] J. Tyson, "Two-sided estimates of minimum-error distinguishability of mixed quantum states via generalized HolevoCurlander bounds", Journal of Mathematical Physics 50, 032106 (2009), arXiv:0907.2094 [quant-ph].

[26] M. A. Ballester, S. Wehner, and A. Winter, "State Discrimination With Post-Measurement Information", IEEE Transactions on Information Theory 54, 4183-4198 (2008), arXiv:quant-ph/0608014.

[27] S. Beigi and A. Gohari, "Quantum Achievability Proof via Collision Relative Entropy”, IEEE Transactions on Information Theory 60, 7980-7986 (2014), arXiv:1312.3822 [quant-ph]. 